\title{
Associations between specific IgE sensitization to 26 respiratory allergen molecules and HLA class II alleles in the EGEA cohort
}

Hubert Gheerbrant ${ }^{1,2^{*}}$, Alicia Guillien ${ }^{2}$, Raphaël Vernet ${ }^{3}$, Christian Lupinek $^{4}$, Christophe Pison $^{1,5}$, Isabelle Pin ${ }^{2,6}$, Florence Demenais ${ }^{3}$, Rachel Nadif ${ }^{7}$, Jean Bousquet ${ }^{8}$, Winfried F Pickl $^{9}$, Rudolf Valenta ${ }^{4,10,11,12}$, Emmanuelle Bouzigon ${ }^{3}$ and Valérie Siroux ${ }^{2}$

${ }^{1}$ Service Hospitalier Universitaire Pneumologie Physiologie, Centre Hospitalier Universitaire Grenoble Alpes, Grenoble, France.

${ }^{2}$ University Grenoble Alpes, Inserm, CNRS, IAB, Team of Environmental Epidemiology Applied to Reproduction and Respiratory Health, 38000 Grenoble, France.

${ }^{3}$ Université de Paris, UMRS 1124, INSERM, Group of Genomic Epidemiology of Multifactorial Diseases, Paris, France.

${ }^{4}$ Division of Immunopathology, Department of Pathophysiology and Allergy Research, Center for Pathophysiology, Infectiology and Immunology, Medical University of Vienna, Austria.

${ }^{5}$ Inserm 1055, Laboratoire de Bioénergétique Fondamentale et Appliquée, Grenoble, France.

${ }^{6}$ Department of Pediatrics, Grenoble-Alpes University Hospital, Grenoble, France.

${ }^{7}$ Université Paris-Saclay, UVSQ, Univ. Paris-Sud, Inserm, Équipe d'Épidémiologie respiratoire intégrative, CESP, 94807, Villejuif, France.

${ }^{8}$ Arnaud de Villeneuve University Hospital and Inserm, Montpellier, France.

${ }^{9}$ Institute of Immunology, Center for Pathophysiology, Infectiology and Immunology, Medical University of Vienna, Vienna, Austria.

${ }^{10}$ NRC Institute of Immunology FMBA of Russia, Moscow, Russia.

${ }^{11}$ Laboratory for Immunopathology, Department of Clinical Immunology and Allergy, Sechenov First Moscow State Medical University, Moscow, Russia. 
${ }^{12}$ Karl Landsteiner University of Health Sciences, Krems, Austria.

\section{Corresponding author}

Hubert Gheerbrant, Service Hospitalier Universitaire Pneumologie Physiologie, Grenoble, France, BP217, 38043 Grenoble Cedex 9, France.

Tel.: +33 645752 994; hgheerbrant@chu-grenoble.fr

\section{Acknowledgments}

The authors thank all those who participated in the setting of the EGEA study and on the examinations of the individuals. We thank the EGEA cooperative group members:

Coordination: V Siroux (epidemiology, PI since 2013); F Demenais (genetics); I Pin (clinical aspects); R Nadif (biology); F Kauffmann (PI 1992-2012).

Respiratory epidemiology: Inserm ex-U 700, Paris: M Korobaeff (Egea1), F Neukirch

(Egea1); Inserm ex-U 707, Paris: I Annesi-Maesano (Egea1-2); Inserm U 1018, Villejuif: O Dumas, F Kauffmann, N Le Moual, R Nadif, MP Oryszczyn (Egea1-2), R Varraso; Inserm U 1209 Grenoble: J Lepeule, V Siroux.

Genetics: Inserm ex-U 393, Paris: J Feingold; Inserm UMR 1124, Paris: E Bouzigon, MH Dizier, F Demenais; CNG, Evry: I Gut (now CNAG, Barcelona, Spain), M Lathrop (now Univ McGill, Montreal, Canada).

Clinical centers: Grenoble: I Pin, C Pison; Lyon: D Ecochard (Egea1), F Gormand, Y Pacheco;Marseille: D Charpin (Egea1), D Vervloet (Egea1-2); Montpellier: J Bousquet; Paris Cochin: A Lockhart (Egea1), R Matran (now in Lille); Paris Necker: E Paty (Egea1-2), P Scheinmann (Egea1-2); Paris-Trousseau: A Grimfeld (Egea1-2), J Just.

Data and quality management: Inserm ex-U155, Paris: J Hochez (Egea1); Inserm U 1018, Villejuif: N Le Moual, L Orsi; Inserm ex-U780, Villejuif: C Ravault (Egea1-2); Inserm ex-U794, Evry: N Chateigner (Egea1-2); Inserm UMR 1124, Paris: H Mohamdi; Inserm U1209, Grenoble: A Boudier, J Quentin (Egea1-2). 
The authors are grateful to the three CIC-Inserm of Necker, Grenoble and Marseille who supported the study and in which participants were examined. They are also grateful to the biobanks in Lille (CIC Inserm), and at Annemasse (Etablissement français du sang) where biological samples are stored. They are indebted to all the individuals who participated, without whom the study would not have been possible. The authors thank the CRISALIS / F-CRIN INSERM network (Clinical Research Initiative in Severe Asthma: a Lever for Innovation \& Science) for the support of some of its members in asthma research.

\section{Funding}

The study was supported in part by ANR-19-CE36-0005-01 NIRVANA, Inserm Aviesan Itmo santé publique, the Scientific committee "AGIR for chronic diseases", grant F4605 of the Austrian Science Fund (FWF), by a grant from the Country of Lower Austria and by the Megagrant of the Government of the Russian Federation, grant number 14.W03.31.0024 to RV and by the European Commission's Seventh Framework Program MeDALL under grant agreement no. 261357. Genotyping was supported grant from the European Commission (No. LSHB-CT-2006-018996-GABRIEL).

\section{Authors contribution}

HG and VS were involved in the conception, hypotheses delineation and design of the analysis; VS, CL, CP, IP, FD, RN, JB, RV, EB and VS participated in the acquisition of data; HG, AG, EB and VS designed and conducted the statistical analysis and interpretation; HG, EB and VS wrote the article; All authors reviewed the manuscript and revised it critically. All authors approved the final version of the manuscript.

\section{Conflict of Interest Statement}

I. Pin receives honaria for presentation from GSK, personal fees from AGIRàdom and receives travel support from SANOFI. R. Valenta reports grants from Country of Lower Austria, grants from Austrian 
Science Fund (FWF), grants from Worg Pharmaceuticals (Hangzhou) Co., Ltd. S Hangzhou, Zhejiang, 310000, China, grants from HVD Biotech, Vienna, Austria, grants and personal fees from Viravaxx, Vienna, Austria, outside the submitted work. C. Lupinek reports personal fees from Thermo Fisher, outside the submitted work. J. Bousquet reports personal fees from Chiesi, Cipla, Hikma, Menarini, Mundipharma, Mylan, Novartis, Purina, Sanofi-Aventis, Takeda, Teva, Uriach, other from KYomedInnov, outside the submitted work. The rest of the authors declare that they have no relevant conflicts of interest. 


\begin{abstract}
Background: Allergy, the most frequent immune disorder affecting $30 \%$ of the world's population, is the consequence of immunoglobin E (IgE) sensitization to allergens. Among the genetic factors suspected to be involved in allergy, the HLA class-II genomic region is a strong candidate.
\end{abstract}

Objective: To assess the association between HLA class-II alleles and specific IgE (sIgE) sensitization to a large number of respiratory allergen molecules.

Methods: The analysis relied on 927 participants of the EGEA cohort, including 497 asthmatics. The study focuses on 26 aeroallergens recognized by sIgE in at least $5 \%$ of the study population (determined with the MEDALL chip with $\operatorname{sIgE} \geq 0.3$ ISU) and 23 imputed HLA class-II alleles. For each sIgE sensitization and HLA class-II allele, we fitted a logistic regression model accounting for familial dependence and adjusted for gender, age and genetic principal components. P-values were corrected for multiple comparisons (False Discovery Rate).

Results: Most of the 19 statistically significant associations observed regard pollen allergens (mugwort Art v1, olive tree Ole e 1, timothy grass $\mathrm{Phl}$ p2, Phl p5 and plantain Pla 11), 3 were mold allergen (Alternaria Alt a 1) and a single one regards house dust mite allergen (Der p7). No association was observed with pet allergens. The strongest associations were found with mugwort Art v1 ( $\mathrm{OR}=5.42$ (95\%CI, $3.30 ; 8.88), 4.14(2.65 ; 6.47), 3.16(1.88 ; 5.31)$ with DQB1*05:01, DQA1*01:01 and DRB1*01:01 respectively).

Conclusion: Our results support the important role of HLA class-II alleles as immune response genes predisposing their carriers for sensitization to various major pollen allergens.

Keywords: genetic epidemiology, HLA class II genes, MeDALL allergen-chip, respiratory allergen, specific $\operatorname{IgE}$ 


\section{Abbreviations}

BMI: Body mass index

CCD: Cross-reactive carbohydrate determinant

CI: Confidence interval

EGEA: Epidemiological study of the Genetics and Environment of Asthma

FDR: False discovery rate

GEE: Generalized Estimating Equation

GWAS: Genome Wide Association Study

HLA: Human leukocyte antigen

IgE: Immunoglobulin E

IQR: Interquartile range

ISU: Standardized units for specific IgE

MEDALL: Mechanisms of the Development of Allergy

MHC: Major histocompatibility complex

OR: Odd ratio

SNP: Single-nucleotide polymorphism 


\section{Introduction}

Allergy is the most important immune disorder affecting $30 \%$ of the world's population [1]. It substantially contributes to the morbidity of asthma, rhinitis and conjunctivitis [2]. Asthma is the leading chronic respiratory disease in the world, affecting nearly 273 million individuals as determined in the year 2017 [3]. All allergic diseases are the consequence of prior allergen-specific sensitization and their manifestations vary depending on the type of allergen and level of exposure.

Allergic sensitization is characterized by the development of high levels of allergen-specific immunoglobulins E (IgE) and IgE-mediated allergic inflammation. Sensitization is a complex process involving both environmental and genetic factors [4]. It is suggested that the host genotype play an important role in the immune response to individual allergens, and the HLA class-II genomic region harbouring the classic immune response genes is a strong candidate. Cell-surface proteins encoded by the HLA class-II genomic region are responsible for the regulation of the immune system in humans, particularly in hypersensitivity diseases such as allergy and autoimmunity. The very high allelic diversity of the HLA system makes it possible to explain the high variability in allergenic sensitization profiles [4]. Marsh et al. identified for the first time an association between genetic polymorphisms of the HLA system (HLA-Dw2) and specific sensitization to ragweed pollen allergens [5]. Since then, several studies supported the important role of the HLA class-II genotypes for predisposition to sensitization against distinct allergens [6-13]. Nevertheless, these association studies were limited by their sample size (only 5 studies included more than 100 individuals). More importantly, by selecting populations on the basis of their sensitization profiles, which were restricted to one or only few specific allergens, these studies were unable to provide a comprehensive overview of the association pattern with a large collection of molecularly defined allergens. In recent years, the advent of Genome Wide Association Study (GWAS) has shifted the focus away from genetic studies of candidate genes. While this pan-genomic approach confirms the involvement of HLA class-II genes in allergy, it does not provide new data on their role [14-16]. In addition, the observations by Fisher et al showing that HLADRB alleles were selectively associated with sIgE to Bet $\mathrm{v} 1$ among individuals monosensitized to Bet $\mathrm{v} 1$, and not among those also sensitized to additional allergens, suggested that HLA class-II alleles 
could play a major role in mono versus polysensitisation [17], an hypothesis that remains to be tested. Finally, allergenic exposure, a necessary condition for sensitization independently of HLA allelic genotype, has rarely been considered in previous studies [18].

The objective of this study was to assess associations between the presence of distinct HLA class-II alleles and the emergence of specific IgE sensitization against a large collection of molecularly-defined respiratory allergens in a large cohort of individuals with a variable degree of allergen exposure. As a secondary objective, we aimed to test whether HLA class-II alleles are preferentially associated with mono versus poly-sensitisation. 


\section{Methods}

\section{Population and study setting}

This analysis relies on participants of the Epidemiological study of the Genetics and Environment of Asthma, bronchial hyper responsiveness and atopy (EGEA), a cohort initiated in the early 1990's in 5 French cities (Paris, Lyon, Grenoble, Montpellier, and Marseille) [18]. In total, 388 asthma cases recruited in chest clinics, 1244 first degree relatives of cases and 415 population-based controls were enrolled (EGEA1: 1991-94). The protocol included, among other things, a clinical exam including lung function, skin prick testing and a blood withdrawal. About 11 years later, 1543 EGEA1 participants $(75.4 \%$, deceased $=45$, refusal or lost $=459)$ accepted to participate in a first follow-up study and 58 new family members were included too (EGEA2: 2004-2007, n=1601). EGEA2 participants replied to a questionnaire and carried out a lung function test, a skin prick test and blood sample collection. The clinical and biological data collection and management were certified ISO 9001 from 2006 to 2018. Written consent was obtained from all individuals. Ethical approval was obtained for both surveys (Cochin Royal Hospital, Paris, for EGEA1 and Necker-Enfants Malades Hospital, Paris, for EGEA2).

Among participants with serum collected at EGEA2, allergen specific IgE (sIgE) data were available for 1006 participants. Samples selected for the determination of allergen sIgE data were randomly picked among individuals with and without a history of asthma (defined as being recruited as an asthma case in chest clinics or by a positive answer to either "Have you ever had attacks of breathlessness at rest with wheezing?" or "Have you ever had asthma attacks?").

\section{Measurement of allergen-specific IgE sensitization}

Allergen sIgE sensitization was determined in anonymized serum samples with the Mechanisms of the Development of Allergy (MeDALL) allergen chip, using sIgE level greater than 0.3 ISU as the cut-off [19]. Overall, sIgE reactivity to 176 allergen components including aero- and food allergen components were assessed [20]. In this study, we focused on 26 aeroallergen sIgEs (house dust mites, pollens, animal dander, fungi) with a sensitization rate in the study population of at least $5 \%$ (for statistical power issue) 
and after exclusion of cross-reactive carbohydrate determinant family (CCD) and highly correlated sIgE $\left(r^{2}>0.9\right)$

Based on the 26 selected respiratory allergens, a 4-class outcome defining mono-, oligo- and polysensitization was built: no-sensitization to any of the 26 selected allergen components; sIgE sensitization to a single allergen component (mono-sensitization); sIgE sensitization to 2-to-4 allergen components (oligo sensitization) and sIgE sensitization to 5 or more allergen components (polysensitization).

\section{Genotyping and imputation of classical HLA class-II alleles}

The genotyping of EGEA individuals was done at the genome-wide level using the Illumina Human 610-Quad array (Illumina, San Diego, CA, USA) as part of the Gabriel asthma Consortium [21]. Among genotyped SNPs, we selected 7,579 SNPs passing the QC criteria (genotype missing rate $<3 \%$, minor allele frequency $\geq 5 \%$ in non-asthmatics and consistency with Hardy-Weinberg equilibrium in nonasthmatics (p-value $\left.>10^{-4}\right)$ ) $[22,23]$ and belonging to the extended major histocompatibility complex (MHC; 20-40 Mb on chromosome 6) in order to impute classical HLA alleles for class II loci at fourdigit resolution using SNP2HLA v1.0 [24] with Type 1 Diabetes Genetics Consortium reference panel, as previously described [25]. A total of 76 alleles was imputed for HLA -DRB1, -DQA1, -DQB1, -DPA1 and-DPB1 loci. Among the 76 alleles, we removed 51 alleles with a frequency less than $5 \%$ and two alleles that were in strong linkage disequilibrium with at least one other allele $\left(r^{2}>0.9\right)$ [26]. A total of 5, 6, 6, 6, 2 and 4 alleles were selected for HLA-DRB1, -DQA1, -DQB1, -DPA1 and -DPB1 loci, respectively. Each allele is considered as a continuous variable coded 0 (absent), 1 (heterozygous) and 2 (homozygous).

In order to conduct sensitivity analysis, imputation of HLA class-II alleles was also performed using HIBAG v1.4, using its hg19 Illumina Human610-Quad-specific European panel available in the HLARES reference population [27]. A total of 79 alleles was imputed for four HLA class-II loci (HLA-DRB1, -DQA1, -DQB1 and -DPB1) with the default setting of HIBAG. Of those, 18 alleles with frequency $\geq 5 \%$ and $\mathrm{r}^{2}<0.9$ were kept for analysis: 3 alleles for HLA-DRB1, 5 for -DQA1, 5 for -DQB1, and 5-DPB1. Among these alleles, 14 were part of the SNP2HLA-selected alleles. We found 
a high level of agreement of imputed HLA alleles between the two methods of imputation (median [IQR] of the Kappa coefficient calculated for each of the 14 alleles $=0.98[0.94-0.99])$.

\section{Statistical analyses}

Principal characteristics of the study population were expressed as percentage or median with interquartile range. For each sIgE sensitization and each HLA class-II allele, we fitted a logistic regression model considering HLA class-II allele as the dependent variable and the allergen sIgE sensitization as the independent variable. Models were adjusted for gender, age and genetic principal components computed using EIGENSTRAT2.0 software to account for population stratification [2830], and the familial dependence of the data was considered ( 927 participants for 487 families) using a mixed model (random intercept model) (R package "GEE"). The above analyses were corrected for multiple comparisons by controlling for the false discovery rate (FDR) [31]. FDR q-values of less than 0.05 were considered statistically significant. Sensitivity analysis was conducted with the HIBAGimputed alleles using best-guess genotypes as predictors, provided they had posterior probability $>0.6$.

Polytomous logistic regression adjusted for age, sex and genetic principal components was conducted to investigate the association between HLA class-II alleles and mono- versus poly-sensitization.

A further analysis was conducted to assess the effect of the ragweed pollen exposure level on the association between Amb a1 sIgE and HLA class-II alleles. Indeed, strong geographic variation of the ragweed pollen exposure exists in France, with exposure being mainly restricted to Lyon area (from measures collected across the year 2006 from the Reseau National de Surveillance Aerobiologique) [18]. We studied the association between HLA class-II alleles and IgE Amb a1 (major ragweed allergen) separately in high and low-exposed regions, respectively in Lyon and Marseille-Montpellier-Paris (the Grenoble region having an intermediate exposure level was not considered in this analysis) and calculated the statistical interaction test.

To address to which extent the asthma status could have affected the findings, we performed a stratified analysis of the statistically significant association on the asthma status. 
Although the regression model (Generalized Estimating Equation, GEE) accounted for the family structure of the data, we performed a sensitivity analysis in a restricted population including only one member of each family to address the robustness of the results to a potential confounding issue related to share exposure levels within families. To maximize the statistical power of this sensitivity analysis, for each family a single individual with asthma and/or rhinitis was primarily selected. A further sensitivity analysis was carried out to address the robustness of our findings in analyses assuming a dominant HLA class-II model.

All analyses were performed using R-3.5.1 software (R Foundation for Statistical Computing, Vienna, Austria).

\section{Results}

\section{Characteristics of the study population}

Data from 927 individuals were analyzed (Paris: $n=258$; Grenoble: $n=289$; Montpellier: $n=62$; Lyon: $\mathrm{n}=145$; Marseille: $\mathrm{n}=173$ ), after exclusion of $79(8 \%)$ participants without GWAS data (Figure 1, Table E-1). The demographic characteristics of individuals are presented in Table 1. The population included of $51 \%$ women and the median age was 38 years [IQR: 25-55]. The study participants belonged to 487 families: 272 of them are including only one individual and 215 are including at least 2 individuals. The distribution of family probands, family members of probands and controls was 237 (26\%), $510(55 \%)$, $180(19 \%)$ individuals, respectively. There was a history of asthma and rhinitis in 54\% $(\mathrm{n}=497)$ and $53 \%$ $(n=489)$ of participants, respectively.

Of the 26 specific IgE antibodies studied, $11(42 \%)$ were directed against indoor allergens (Dermatophagoides Der p 1, Der p 4, Der p 5, Der p 7, Der p 10, Der p 18, Der p 21, Der p 23, Der p 27, Der f 2 and Blomia Tropicalis Blo t 5), 10 (39\%) were directed against pollen allergens (alder Aln g 1, mugwort Art v 1, birch Bet v 1, olive Ole e 1, timothy grass Phl p 1, Phl p 2, Phl p 5, Phl p 6, Phl p 11 and plane tree Pla I 1), four (15\%) were directed against animals allergens (dog Can f 1, Can $\mathrm{f} 5$ and cat Fel d 1, Fel d 4) and one was directed against a mould allergen (Alternaria Alt a 1). SIgE sensitization varied from 5\% for Blo t 5, Der p 18 and Fel d 4 to 35\% for Phl p 1 (Table 1). 


\section{HLA class-II alleles and allergen-specific IgE sensitization}

The associations between sIgE responses to 26 respiratory-allergens and 23 SNP2HLA imputed HLA class-II alleles were tested, leading to 598 tests (Figure E-1). Nineteen associations were statistically significant after controlling for multiple comparisons (Table 2). Most of the statistically significant associations (15 over the 19) were observed with pollen allergens (olive tree Ole e 1, mugwort Art v 1, birch Bet v 1, timothy grass Phl p 2, Phl p 5 and plantain Pla 11), three were observed with Alternaria Alt a 1 and a single one was observed with a house dust mite allergen component (Der $\mathrm{p} 7$ ). The strongest effects were found for mugwort Art v 1 (associated with DQB1*05:01, DQA1*01:01 and DRB1*01:01 with $\mathrm{OR}=5.42(95 \% \mathrm{CI}, 3.30 ; 8.88), 4.14$ (2.65;6.47), $3.16(1.88 ; 5.31)$, respectively). No significant association was detected for any allergic sensitization to animal allergens.

Sensitivity analysis performed with HIBAG imputed alleles detected 13 statistically significant associations after controlling for multiple testing. Of these, 11 were common to the SNP2HLA data with comparable OR's magnitude. The two additional statistically significant associations revealed by HIBAG regard allergens already found to be associated with HLA class-II alleles with SNP2HLA (Ole e 1 and Alt a 1) (Table 2).

Sensitivity analysis conducted on the sub-population consisting of a single individual per family ( $\mathrm{n}=487$ ), showed consistent findings, with ORs of very similar magnitude (Table $\mathbb{E}-2$ ). The sensitivity analysis on a dominant expression of the HLA class-II alleles showed similar findings, with statistically significant associations observed almost exclusively between HLA class-II alleles and sIgE to pollen allergens. Nevertheless, a general trend was observed for ORs of stronger magnitude with the dominant genetic model as compared to the co-dominant model. (Table E-3).

The analysis stratified on the asthma status showed ORs of the same order of magnitude in individuals with and without asthma. (Figure 2). However, given the lower statistical power (related to lower prevalence of $\operatorname{sgE}$ ) in individuals without asthma than in individuals with asthma, less associations were statistically significant in individuals without asthma than in those with asthma (10 vs. 19). 


\section{HLA class-II alleles and monosensization versus polysensitization}

Overall, for each HLA class-II allele, the associations observed with polysensitization and monosensitization were of similar magnitude (Table 3 ). Only one allele (DRB1*11:01) showed a borderline statistically significant increased risk of poly-versus mono-sensitization (OR=2.27 95\% CI: $0.99-5.24)$.

\section{Associations between sIgE to the major ragweed allergen Amb a 1 and HLA class-II alleles stratified by the level of ragweed exposure.}

In 2006, the city the most exposed to ragweed pollen was Lyon with an average of more than 10 grains/m3 per day. Montpellier, Marseille and Paris were only slightly exposed with an average of less than 2 grains/m3 per day. IgE recognition to Amb a 1 was found for 35 individuals (4\%), among which 22 were from Lyon (63\%). Three HLA class-II alleles (DRB1*03:01, DQB1*02:01 and DQA1*05:01) were statistically significantly associated with Amb a1 IgE sensitization among the participants from Lyon, with OR estimates varying from 2.0 to 4.0, while no association was observed for these HLA class-II alleles in individuals from Paris-Montpellier-Marseille, with OR estimates varying from 1.0 to 1.5 (Table 4). However, the interaction tests were statistically significant for one interaction (DPB1*04:01), with an $\mathrm{OR}=0.26(95 \% \mathrm{CI}: 0.12-0.57)$ among individuals from Paris-MontpellierMarseille versus 1.58 (95\% CI: 1.15-2.17) among individuals from Lyon (interaction p-value=0.03). 


\section{Discussion}

To our knowledge, this study is the first to address the association between the HLA class-II alleles and allergen-specific IgE sensitization to a large set of allergen components in about 1000 individuals. Our results revealed 19 statistically significant associations almost exclusively with pollen allergen molecules. None of the HLA class-II allele was specifically associated with monosensitization. Overall, our study confirms the role of HLA class-II genes in the development of allergic sensitization and suggests that these genes might be specifically associated with sensitization to pollen allergens.

The strongest associations found in our study concern the major pollen allergen from mugwort. In fact, sIgE sensitization against the major mugwort pollen allergen Art v 1 was significantly associated with the presence of the DR1 haplotype consisting of DQA1*01:01 and DQB1*05:01, and DRB1*01:01, regardless of the HLA imputation method used. This finding confirms previous observations by JahnSmith et al. and Torio et al. who reported an association between sensitization against the mugwort Art v 1 allergen and the allelic group HLA-DRB $1 * 01$ and HLA-DQB $1 * 05: 01$ with similar OR as those detected in the present study [7,13]. The importance of the HLA-DRB1 gene for allergic sensitization was further supported by the in vitro study performed by B. Knapp et al. who demonstrated that two alleles of this gene (01:01 and 04:01) modified the three-dimensional conformation of the respective HLA class-II protein, and by the in vivo studies of Neunkirchner et al. who have formally proven that the HLA-DRB $1 * 01: 01$ allele when expressed in mice makes them significantly more susceptible to aerosol challenge with mugwort pollen extract than their wildtype litters [32]. Thus, the presentation of Art v 1 to T-cell receptors differed according to the HLA class-II protein involved which consequently modified the risk of lymphocyte activation [6].

Our study also identified associations between alleles in HLA class-II and olive tree-derived Ole e 1 sIgE. This finding converges with a previous study by Cárdaba et al. that showed an association with HLA DQB1*02:01 (DQ2 haplotype) of similar magnitude (OR=2.64; 95\% CI: 2.01;3.46) [11]. The same research group subsequently showed in vitro the inhibition of the proliferative response of T-cell lines specific to Ole e I with HLA-DQ2 antibodies [33]. This confirms the restriction of specific T proliferation by HLA class-II molecule in allergy. 
The literature suggested associations between HLA class-II alleles and the risk of allergic sensitization to house dust mite and pets' allergens [8-10]. However, although half of the allergen s-IgE sensitization tested in our study regards house dust mite and pets' allergen, we did not identify any statistically significant association with allergic sensitization to pets' allergens, and a single negative association was observed for house dust mite allergen. The prevalence of sensitization to Der $\mathrm{p} 1$, a major mite allergen, was high in our population (27\%) and higher than for Art v 1 (9\%) or Bet v $1(10 \%)$. Therefore the lack of association does not result from a lower statistical power for theses allergens as compared with pollens. One hypothesis for the observed differences in the associations of HLA class-II alleles with sIgE to pollen allergens and perennial allergens relates to the fact that for allergen sources such as mites, which contain several major allergens with each allergen harbouring more than one immunodominant $\mathrm{T}$ cells epitope, it might be more difficult to assign a single HLA allele which is taking the lead during the sensitization process $[34,35]$. Another hypothesis relates to previous observations showing different patterns of $\operatorname{IgE}$ recognition frequency to respiratory allergens, with $\operatorname{IgE}$ recognition to pollen allergen preferentially associated with rhinitis and $\operatorname{IgE}$ recognition to perennial allergens (mites, pets) preferentially associated with asthma [36]. Therefore, one could suggest that among the various genes involved in allergic diseases, HLA class-II genes may play a major role in the development of allergic sensitization to pollen allergens that are more specifically associated with the development of rhinitis. In a recent GWAS on allergic rhinitis, the HLA genomic region contained the strongest association, including independent signals located around HLA-DQB1 gene [37].

Overall, our findings do not support the hypothesis that HLA class-II alleles could be preferentially associated with mono vs polysensitization, as suggested by the observations from Fisher et al [17]. Actually, our results showing associations of some HLA class-II alleles with sensitization to different allergens from distinct allergen families, would go the other way around. Indeed, DQA1*02:01 was associated with an increased risk for sIgE sensitization to the major Alternaria allergen Alt al (no family protein), the olive tree Ole e 1 (common olive group 1) and thimothy grass Phl p 5 (undefined protein group). This suggests that this specific HLA Class-II DQA1 allele might actually favour the 
development of polysensitization above the mechanisms of polysensitization that results from the molecular mimicry of allergens from the same protein family [38,39]. In fact, the nomenclature of the HLA class II alleles is random and does not account for the architecture (tertiary and quaternary) structure of the HLA-class II molecules, in particular, the "binding groove" for immunogenic peptides. We can assume that DQA1*02:01 contains a "super-structure", encoded by a short nucleotide sequence and constituting a high affinity anchor site for immunogenic peptides found in various allergenic molecules. A "super-structure" was highlighted by Fischer et al. which was significantly associated with the reactivity of Bet v 1 [17]. Further studies should consider grouping HLA alleles according to their primary biological function rather than by the name of the allele [40]. Finally, in a recent study, a significant association was shown between the HLA-DQB1 loci and asthma severity [41]. Interestingly, 5 of the 19 significant associations observed in our study regard HLA-DQB1 alleles. Altogether these observations might suggest that the severity of asthma and SIgE sensitization share common HLA classII genetic variants or that the severity of asthma is partly mediated by $\operatorname{sIgE}$ sensitization.

Although HLA class-II genes were found to be associated with asthma [21,41], we did not consider asthma as an adjustment variable. Our hypothesis is that the association reported between HLA class-II genes and asthma is partly mediated by specific IgE sensitization, asthma being a consequence of sIgE sensitization rather than a cause [4]. The similarity of the association pattern in individuals with and without asthma suggests that the association reported between HLA class-II genes and sIgE sensitization is independent of the asthma status.

One of the main strengths of this study relates to the study population. Indeed, by analysing data of about 1000 participants from the EGEA cohort, makes it the largest study of its kind. Furthermore, this study, including $54 \%$ of individuals with asthma, thus with a high allergic sensitisation rate, was able to address associations between HLA class-II alleles and IgE responses to 26 important respiratory allergen molecules with a good statistical power. Our results are supported by sensitivity analysis. In particular although the statistical model accounted for the family structure of the data, the analysis conducted in a sub-population including only one member per family led to the same conclusion, ensuring that the family design of the study did not led to confounding issues through shared environment within families. 
Moreover, our main findings persist when considering a dominant genetic model of HLA class II alleles. An additional asset relies on the accurate and wide characterization of $\operatorname{IgE}$ sensitisation, by assessing sIgE to a large set of allergen molecules using a validated micro-array assay [18].

A potential limitation of our analysis is that we did not consider the allergen exposure, although this is a necessary condition for allergen sensitization. In our study in adults, that covers a large range of aeroallergens, accurately assessing exposure to each allergen source since childhood, a critical window of time in the development of allergic sensitization, is almost impossible. We showed that the magnitude of the associations between ragweed Amb a 1 and some HLA class-II alleles (e.g. drb1*03:01) tended to be stronger in participants who were a priori more exposed to ragweed pollens (Lyon) as compared to the estimated association in the whole population. This suggests that for allergens with heterogeneous exposure among the EGEA participants, not considering allergenic exposure may lead to underestimated risks between the HLA class-II allele and sIgE, but could not explain the highlighted associations. It may also partly explain why no association between Bet v1 and HLA-DRB3*01:01 [12] was observed in the EGEA study because it includes few patients from the north of France where birch exposure is the highest (the prevalence of sensitization to Bet v1, a major birch allergen, is $10 \%$ in our population while it is more than $20 \%$ in the Swedish BAMSE cohort [42]). Nevertheless, for most of the allergen studied, the exposure is quite common in France and most of the participants should have encountered these allergens over their life. This is particularly true for perennial and ubiquitous allergens molecules from animals and mites. A further limitation of our study lies in the fact that HLA class-II alleles were imputed, a method associated to a lower accuracy as compared with the nucleotide sequencing techniques. However, these imputation methods are becoming more and more efficient and we found strong concordance in the results based on two different imputation methods (HIBAG and SNP2HLA).

\section{Conclusion}

This study underlines the important role of the HLA class-II alleles in allergen-specific IgE sensitization, and more specifically to pollens allergen molecules. By showing that some HLA class-II alleles are associated with sensitization to different allergens from different allergen families, this study indicates 
that HLA class-II alleles may favour the development of polysensitization above the mechanisms of polysensitization that results from the molecular mimicry of allergens from the same protein family.

\section{References}

1. Valenta R, Ferreira F, Focke-Tejk1 M et al. From allergen genes to allergy vaccines. Annu Rev Immunol 2010;28:211-41.

2. Anto JM, Bousquet J, Akdis $\mathrm{M}$ et al. Mechanisms of the Development of Allergy (MeDALL): Introducing novel concepts in allergy phenotypes. J Allergy Clin Immunol 2017;139:388-99.

3. GBD 2017 Disease and Injury Incidence and Prevalence Collaborators. Global, regional, and national incidence, prevalence, and years lived with disability for 354 diseases and injuries for 195 countries and territories, 1990-2017: a systematic analysis for the Global Burden of Disease Study 2017. Lancet 2018;392:1789-858.

4. Valenta R, Karaulov A, Niederberger V et al. Molecular Aspects of Allergens and Allergy. Adv Immunol 2018;138:195-256.

5. Marsh DG, Hsu SH, Roebber M et al. HLA-Dw2: a genetic marker for human immune response to short ragweed pollen allergen Ra5. I. Response resulting primarily from natural antigenic exposure. J Exp Med 1982;155:1439-51.

6. Knapp B, Fischer G, Hemelen DV al. Association of HLA-DR1 with the allergic response to the major mugwort pollen allergen: molecular background. BMC Immunol 2012;13:1-11.

7. Jahn-Schmid B, Fischer GF, Bohle B et al. Antigen presentation of the immunodominant T-cell epitope of the major mugwort pollen allergen, Art $v 1$, is associated with the expression of HLADRB1 *01. J Allergy Clin Immunol 2005;115:399-404.

8. $\mathrm{Hu}$ C, Hsu PN, Lin RH et al. HLA DPB $1 * 0201$ allele is negatively associated with immunoglobulin E responsiveness specific for house dust mite allergens in Taiwan. Clin Exp Allergy 2000;30:538-45.

9. Young RP, Dekker JW, Wordsworth BP et al. HLA-DR and HLA-DP genotypes and immunoglobulin E responses to common major allergens. Clin Exp Allergy 1994;24:431-9.

10. Lara-Marquez ML, Yunis JJ, Layrisse $Z$ et al. Immunogenetics of atopic asthma: association of DRB1*1101 DQA1*0501 DQB1*0301 haplotype with Dermatophagoides spp.-sensitive asthma in a sample of the Venezuelan population. Clin Exp Allergy 1999;29:60-71.

11. Cárdaba B, Vilches C, Martín E et al. DR7 and DQ2 are positively associated with immunoglobulin-E response to the main antigen of olive pollen (Ole e I) in allergic patients. Hum Immunol 1993;38:293-9.

12. Sparholt SH, Georgsen J, Madsen HO et al. Association between HLA-DRB $3 * 0101$ and immunoglobulin-E responsiveness to Bet v I. Hum Immunol 1994;39:76-8.

13. Torío A, Sánchez-Guerrero I, Muro M et al. HLA class II genotypic frequencies in atopic asthma: association of DRB1*01-DQB1*0501 genotype with Artemisia vulgaris allergic asthma. Hum Immunol 2003;64:811-5. 
14. Schoettler N, Rodríguez E, Weidinger $\mathrm{S}$ et al. Advances in asthma and allergic disease genetics: Is bigger always better? J Allergy Clin Immunol 2019;144:1495-506.

15. Vince N, Limou S, Daya $M$ et al. Association of HLA-DRB1*09:01 with tIgE levels among African-ancestry individuals with asthma. J Allergy Clin Immunol 2020; 146:147-157.

16. El-Husseini ZW, Gosens R, Dekker F et al. The genetics of asthma and the promise of genomicsguided drug target discovery. Lancet Respir Med 2020; 8:1045-1056.

17. Fischer GF, Pickl WF, Faé I et al. Association between IgE response against Bet v I, the major allergen of Birch Pollen, and HLA-DRB alleles. Hum Immunol 1992;33:259-65.

18. Siroux V, Lupinek C, Resch Y et al. Specific IgE and IgG measured by the MeDALL allergenchip depend on allergen and route of exposure: The EGEA study. J Allergy Clin Immunol 2017;139:643-654.e6.

19. Westman M, Lupinek C, Bousquet J et al. Early childhood IgE reactivity to pathogenesis-related class 10 proteins predicts allergic rhinitis in adolescence. J Allergy Clin Immunol 2015;135:11991206.e1-11.

20. Lupinek C, Wollmann E, Baar A et al. Advances in allergen-microarray technology for diagnosis and monitoring of allergy: the MeDALL allergen-chip. Methods San Diego Calif 2014;66:106-19.

21. Moffatt MF, Gut IG, Demenais F et al. A large-scale, consortium-based genomewide association study of asthma. N Engl J Med 2010;363:1211-21.

22. Imboden M, Bouzigon E, Curjuric I et al. Genome-wide association study of lung function decline in adults with and without asthma. $J$ Allergy Clin Immunol 2012;129(5):1218-28.

23. Sarnowski C, Sugier P-E, Granell R et al. Identification of a new locus at $16 \mathrm{q} 12$ associated with time to asthma onset. J Allergy Clin Immunol 2016;138(4):1071-80.

24. Jia X, Han B, Onengut-Gumuscu S et al. Imputing amino acid polymorphisms in human leukocyte antigens. PloS One 2013;8:e64683.

25. Smit LAM, Strachan DP, Vermeulen R et al. Human leukocyte antigen class II variants and adultonset asthma: does occupational allergen exposure play a role? Eur Respir J 2014;44:1234-42.

26. VanLiere JM, Rosenberg NA. Mathematical properties of the $\mathrm{r} 2$ measure of linkage disequilibrium. Theor Popul Biol 2008;74:130-7.

27. Zheng $\mathrm{X}$, Shen J, Cox $\mathrm{C}$ et al. HIBAG--HLA genotype imputation with attribute bagging. Pharmacogenomics $J$ 2014;14:192-200.

28. Shah R, Newcomb DC. Sex Bias in Asthma Prevalence and Pathogenesis. Front Immunol 2018;9.

29. McVean G. A genealogical interpretation of principal components analysis. PLoS Genet 2009;5:e1000686.

30. Price AL, Patterson NJ, Plenge RM, Weinblatt ME, Shadick NA, Reich D. Principal components analysis corrects for stratification in genome-wide association studies. Nat Genet 2006;38(8):904-9.

31. Benjamini $Y$, Drai D, Elmer G et al. Controlling the false discovery rate in behavior genetics research. Behav Brain Res 2001;125:279-84. 
32. Neunkirchner A, Kratzer B, Köhler C et al. Genetic restriction of antigen-presentation dictates allergic sensitization and disease in humanized mice. EBioMedicine 2018;31:66-78.

33. Cárdaba B, De Pablo R, Vilches $\mathrm{C}$ et al. Allergy to olive pollen: T-cell response from olive allergic patients is restricted by DR7-DQ2 antigens. Clin Exp Allergy 1996;26:316-22.

34. Huang H-J, Curin M, Banerjee $\mathrm{S}$ et al. A hypoallergenic peptide mix containing $\mathrm{T}$ cell epitopes of the clinically relevant house dust mite allergens. Allergy 2019;74:2461-78.

35. Posa D, Perna S, Resch $\mathrm{Y}$ et al. Evolution and predictive value of IgE responses toward a comprehensive panel of house dust mite allergens during the first 2 decades of life. $J$ Allergy Clin Immunol 2017;139:541-549.e8.

36. Siroux V, Ballardini N, Soler M et al. The asthma-rhinitis multimorbidity is associated with IgE polysensitization in adolescents and adults. Allergy 2018;73:1447-58.

37. Waage J, Standl M, Curtin JA et al. Genome-wide association and HLA fine-mapping studies identify risk loci and genetic pathways underlying allergic rhinitis. Nat Genet 2018;50:1072-80.

38. Westman M, Lupinek $\mathrm{C}$, Bousquet $\mathrm{J}$ et al. Early childhood $\operatorname{IgE}$ reactivity to pathogenesis-related class 10 proteins predicts allergic rhinitis in adolescence. J Allergy Clin Immunol 2015;135:11991206.e11.

39. Yamamoto K, Ishibashi O, Sugiura K et al. Crystal structure of the dog allergen Can $\mathrm{f} 6$ and structure-based implications of its cross-reactivity with the cat allergen Fel d 4. Sci Rep 2019;9:1503.

40. Bondinas GP, Moustakas AK, Papadopoulos GK. The spectrum of HLA-DQ and HLA-DR alleles, 2006: a listing correlating sequence and structure with function. Immunogenetics 2007;59:539-53.

41. Yan Q, Forno E, Herrera-Luis E et al. A genome-wide association study of asthma hospitalizations in adults. $J$ Allergy Clin Immunol 2020. Online ahead of print.

42. Wickman M, Lupinek C, Andersson N et al. Detection of IgE Reactivity to a Handful of Allergen Molecules in Early Childhood Predicts Respiratory Allergy in Adolescence. EBioMedicine 2017;26:91-9. 


\section{Tables}

TABLE 1. Characterization of the study population

\begin{tabular}{|c|c|c|c|}
\hline \multirow{2}{*}{$\begin{array}{c}\text { Characteristic } \\
\text { Female gender }\end{array}$} & & \multicolumn{2}{|c|}{ n (\%) / median [IQR] } \\
\hline & & 472 & $(51)$ \\
\hline \multicolumn{2}{|l|}{ Age (years) } & 38 & {$[25 ; 55]$} \\
\hline \multicolumn{2}{|c|}{ BMI $\left(\mathrm{kg} / \mathrm{m}^{2}\right)(\mathrm{MD}=16)$} & 23 & {$[21 ; 26]$} \\
\hline \multicolumn{4}{|c|}{ Recruitment center } \\
\hline \multicolumn{2}{|l|}{ Grenoble } & 289 & $(31)$ \\
\hline \multicolumn{2}{|l|}{ Lyon } & 145 & $(16)$ \\
\hline \multicolumn{2}{|l|}{ Marseille } & 173 & (19) \\
\hline \multicolumn{2}{|l|}{ Montpellier } & 62 & (7) \\
\hline \multicolumn{2}{|l|}{ Paris } & 258 & $(28)$ \\
\hline \multicolumn{2}{|l|}{ Asthma ever } & 497 & $(54)$ \\
\hline \multicolumn{2}{|l|}{ Current asthma* } & 417 & $(84)$ \\
\hline \multicolumn{4}{|c|}{ Age of asthma diagnosis $(\mathrm{MD}=32)$} \\
\hline \multicolumn{2}{|c|}{$\leq 4$ years } & 149 & (16) \\
\hline \multicolumn{2}{|l|}{$4-16$ years } & 165 & $(18)$ \\
\hline \multicolumn{2}{|l|}{$>16$ years } & 151 & $(16)$ \\
\hline \multicolumn{2}{|c|}{ History of childhood respiratory infection } & 200 & $(22)$ \\
\hline \multicolumn{2}{|l|}{ Eczema ever } & 347 & (37) \\
\hline \multicolumn{2}{|c|}{ Allergic rhinitis ever $(\mathrm{MD}=13)$} & 489 & $(53)$ \\
\hline \multicolumn{2}{|c|}{ Total IgE (IU/mL), GM } & 87.1 & {$[28.8 ; 213.8]$} \\
\hline \multicolumn{4}{|c|}{ Number of sIgE sensibilized (MD=18) } \\
\hline \multicolumn{2}{|l|}{0} & 381 & (41) \\
\hline \multicolumn{2}{|l|}{1} & 67 & (7) \\
\hline \multicolumn{2}{|l|}{2 to 5} & 126 & (14) \\
\hline \multicolumn{2}{|l|}{$\geq 5$} & 335 & (36) \\
\hline \multicolumn{4}{|c|}{ Allergen specific $\operatorname{IgE}^{\dagger}$} \\
\hline Alder & Aln $g 1$ & 67 & (7) \\
\hline Altenaria & Alt a 1 & 86 & (9) \\
\hline Mugwort & Art v 1 & 58 & (6) \\
\hline Birch & Bet $v 1$ & 95 & $(10)$ \\
\hline Storage mite & Blo t 5 & 50 & $(5)$ \\
\hline Plantain & Pla 11 & 54 & (6) \\
\hline Olive & Ole e 1 & 191 & $(21)$ \\
\hline \multirow[t]{5}{*}{ Timothy grass } & $\mathrm{Phl} \mathrm{p} 1$ & 324 & $(35)$ \\
\hline & $\mathrm{Phl} \mathrm{p} 2$ & 157 & (17) \\
\hline & $\mathrm{Phl}$ p 5 & 175 & (19) \\
\hline & $\mathrm{Phl} \mathrm{p} 6$ & 127 & (14) \\
\hline & Phl p 11 & 64 & (7) \\
\hline House dust mite & Der f 2 & 290 & (31) \\
\hline
\end{tabular}




$\begin{array}{llrl} & \text { Der p 1 } & 252 & (27) \\ \text { Der p 4 } & 129 & (14) \\ & \text { Der p 5 (MD=18) } & 163 & (18) \\ & \text { Der p 7 } & 161 & (17) \\ & \text { Der p 10 } & 133 & (14) \\ & \text { Der p 18 } & 47 & (5) \\ & \text { Der p 21 } & 114 & (12) \\ & \text { Der p 23 } & 260 & (28) \\ & \text { Der p 37 } & 76 & (8) \\ & \text { Fel d 1 } & 231 & (25) \\ \text { Cat } & \text { Fel d 4 } & 50 & (5) \\ & \text { Can f 1 } & 58 & (6) \\ & \text { Can f 5 } & 104 & (11)\end{array}$

HLA class-II allele ${ }^{\ddagger}$

\begin{tabular}{|c|c|}
\hline DRB1*01:01 & $82 \% / 17 \% / 1 \%$ \\
\hline DRB1*03:01 & $81 \% / 18 \% / 1 \%$ \\
\hline DRB1*04:01 & $88 \% / 12 \% / 0 \%$ \\
\hline DRB1*11:01 & $83 \% / 16 \% / 1 \%$ \\
\hline DRB1*15:01 & $81 \% / 18 \% / 1 \%$ \\
\hline DQA1*01:01 & $72 \% / 26 \% / 2 \%$ \\
\hline DQA1*01:02 & $70 \% / 27 \% / 3 \%$ \\
\hline DQA1*01:03 & $85 \% / 14 \% / 1 \%$ \\
\hline DQA1*02:01 & $71 \% / 26 \% / 3 \%$ \\
\hline DQA1*03:01 & $74 \% / 24 \% / 2 \%$ \\
\hline DQA1*05:01 & $52 \% / 40 \% / 8 \%$ \\
\hline DQB1*02:01 & $60 \% / 34 \% / 6 \%$ \\
\hline DQB1*03:01 & $62 \% / 33 \% / 5 \%$ \\
\hline DQB1*03:02 & $83 \% / 16 \% / 1 \%$ \\
\hline DQB1*05:01 & $78 \% / 21 \% / 1 \%$ \\
\hline DQB1*06:02 & $84 \% / 16 \% / 0 \%$ \\
\hline DQB1*06:03 & $86 \% / 13 \% / 1 \%$ \\
\hline DPA $1 * 01: 03$ & $61 \% / 34 \% / 5 \%$ \\
\hline DPA1*02:01 & $68 \% / 29 \% / 3 \%$ \\
\hline DPB1*02:01 & $74 \% / 24 \% / 2 \%$ \\
\hline DPB1*03:01 & $81 \% / 18 \% / 1 \%$ \\
\hline DPB1*04:01 & $39 \% / 46 \% / 15 \%$ \\
\hline DPB1*04:02 & $79 \% / 20 \% / 1 \%$ \\
\hline
\end{tabular}

* current asthma defined among participants with asthma ever by asthma attacks or use of asthma treatment in the past 12 months

${ }^{\dagger}$ Positive allergen-specific IgE defined with a threshold of 0.3 ISU IgE

$\ddagger$ Allele prevalence (\% absent / \% heterozygous / \% homozygous)

BMI: Body mass index; HLA: Human leukocyte antigen; IgE: Immunoglobulin E; MD: missing data 
TABLE 2. Significant associations of respiratory allergen-specific IgE sensitization with HLA class-II alleles (FDR $\leq 0.05$ )

\begin{tabular}{|c|c|c|c|c|c|c|c|c|c|c|c|}
\hline \multirow[b]{2}{*}{$\begin{array}{l}\text { Allergen } \\
\text { component }\end{array}$} & \multirow[b]{2}{*}{$\begin{array}{c}\text { HLA class II } \\
\text { Allele }\end{array}$} & \multicolumn{5}{|c|}{ SNP2HLA imputation } & \multicolumn{5}{|c|}{ HIBAG imputation } \\
\hline & & $\begin{array}{l}\text { Frequency of } \\
\text { IgE recognition } \\
\text { in the presence } \\
\text { of respective } \\
\text { HLA allele }\end{array}$ & $\begin{array}{l}\text { Frequency of } \\
\text { IgE recognition } \\
\text { in the absence } \\
\text { of respective } \\
\text { HLA allele }\end{array}$ & OR & CI 95\% & q-value ${ }^{\dagger}$ & $\begin{array}{l}\text { Frequency of } \\
\text { IgE recognition } \\
\text { in the presence } \\
\text { of respective } \\
\text { HLA allele }\end{array}$ & $\begin{array}{l}\text { Frequency of } \\
\text { IgE recognition } \\
\text { in the absence } \\
\text { of respective } \\
\text { HLA allele }\end{array}$ & OR & CI 95\% & q-value ${ }^{\dagger}$ \\
\hline Alt a 1 & DQA1*01:01 & 0.04 & 0.11 & 0.35 & $(0.20-0.63)$ & 0.02 & NA & NA & NA & NA & NA \\
\hline Alt a 1 & DQA1*02:01 & 0.16 & 0.06 & 2.57 & $(1.76-3.76)$ & $4.65 \mathrm{E}-05$ & 0.16 & 0.07 & 2.46 & $(1.66-3.65)$ & $3.67 \mathrm{E}-04$ \\
\hline Alt a 1 & DQB1*05:01 & 0.04 & 0.11 & 0.32 & $(0.16-0.63)$ & 0.05 & 0.04 & 0.11 & 0.34 & $(0.17-0.67)$ & 0.07 \\
\hline Art v 1 & DPA1*01:03 & 0.11 & 0.04 & 2.57 & $(1.65-4.00)$ & $9.50 \mathrm{E}-03$ & NA & NA & NA & NA & NA \\
\hline Art v 1 & DPA1*02:01 & 0.11 & 0.04 & 2.40 & $(1.52-3.78)$ & 0.02 & NA & NA & NA & NA & NA \\
\hline Art v 1 & DQA1*01:01 & 0.15 & 0.03 & 4.14 & $(2.65-6.47)$ & $3.79 \mathrm{E}-08$ & NA & NA & NA & NA & NA \\
\hline Art v 1 & DQB1*05:01 & 0.19 & 0.03 & 5.42 & $(3.30-8.88)$ & $3.63 \mathrm{E}-07$ & 0.19 & 0.03 & 4.95 & $(2.98-8.22)$ & $1.17 \mathrm{E}-05$ \\
\hline Art v 1 & DRB1*01:01 & 0.15 & 0.04 & 3.16 & $(1.88-5.31)$ & $3.02 \mathrm{E}-03$ & 0.19 & 0.05 & 3.20 & $(1.82-5.63)$ & 0.01 \\
\hline Bet v 1 & DQB1*06:02 & 0.18 & 0.09 & 2.36 & $(1.47-3.79)$ & 0.04 & NA & NA & NA & NA & NA \\
\hline Bet v 1 & DRB1*15:01 & 0.17 & 0.09 & 2.39 & $(1.52-3.77)$ & 0.02 & 0.18 & 0.10 & 2.06 & $(1.24-3.43)$ & 0.16 \\
\hline Der p 7 & DPB1*04:01 & 0.15 & 0.21 & 0.64 & $(0.48-0.84)$ & 0.02 & 0.15 & 0.23 & 0.62 & $(0.46-0.84)$ & 0.04 \\
\hline Ole e 1 & DQA1*02:01 & 0.30 & 0.17 & 2.03 & $(1.50-2.76)$ & $2.01 \mathrm{E}-04$ & 0.30 & 0.16 & 2.04 & $(1.48-2.80)$ & $6.86 \mathrm{E}-04$ \\
\hline Ole e 1 & DQB1*02:01 & 0.32 & 0.13 & 2.64 & $(2.01-3.46)$ & $4.84 \mathrm{E}-09$ & 0.37 & 0.17 & 2.81 & $(1.92-4.10)$ & $2.76 \mathrm{E}-05$ \\
\hline Ole e 1 & DQB1*03:01 & 0.14 & 0.24 & 0.47 & $(0.33-0.67)$ & $1.72 \mathrm{E}-03$ & 0.15 & 0.24 & 0.46 & $(0.32-0.68)$ & $2.87 \mathrm{E}-03$ \\
\hline Ole e 1 & DRB1*03:01 & 0.36 & 0.17 & 2.69 & $(1.88-3.83)$ & $1.24 \mathrm{E}-05$ & NA & NA & NA & NA & NA \\
\hline Phl p 2 & DQA1*05:01 & 0.23 & 0.12 & 1.86 & $(1.40-2.45)$ & $1.02 \mathrm{E}-03$ & NA & NA & NA & NA & NA \\
\hline Phl p 2 & DRB1*03:01 & 0.28 & 0.14 & 2.10 & $(1.44-3.07)$ & 0.01 & NA & NA & NA & NA & NA \\
\hline Phl p 5 & DQA1*02:01 & 0.24 & 0.17 & 1.63 & $(1.20-2.23)$ & 0.05 & 0.24 & 0.17 & 1.69 & $(1.22-2.34)$ & 0.04 \\
\hline Pla 11 & DPB1*04:02 & 0.15 & 0.03 & 2.91 & $(1.75-4.83)$ & $5.07 \mathrm{E}-04$ & 0.15 & 0.03 & 3.11 & $(1.88-5.15)$ & $2.75 \mathrm{E}-04$ \\
\hline
\end{tabular}

$\dagger$ FDR-controlling procedures

CI: Confidence Interval; NA: Imputed alleles not available; OR: Odd Ratio

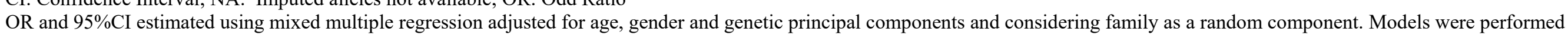
assuming an additive genetic model.

TABLE 3. Association between mono- and poly-sensitization and HLA class-II alleles

Allele 1 sIgE+ vs. 0 sIgE+




\begin{tabular}{|c|c|c|c|c|c|c|c|c|c|}
\hline & OR & IC 95\% & p-value & OR & IC $95 \%$ & p-value & OR & IC $95 \%$ & p-value \\
\hline DRB1*01:01 & 0.71 & $0.36-1.42$ & 0.34 & 0.67 & $0.44-1.02$ & 0.06 & 0.94 & $0.46-1.92$ & 0.87 \\
\hline DRB1*03:01 & 2.37 & $1.35-4.16$ & $2.61 \mathrm{E}-03$ & 1.95 & $1.29-2.94$ & 1.53E-03 & 0.82 & $0.47-1.42$ & 0.48 \\
\hline DRB1*04:01 & 0.55 & $0.23-1.32$ & 0.18 & 0.74 & $0.46-1.18$ & 0.20 & 1.33 & $0.55-3.25$ & 0.52 \\
\hline DRB1*11:01 & 0.73 & $0.32-1.67$ & 0.46 & 1.67 & $1.07-2.59$ & 0.02 & 2.27 & $0.99-5.24$ & 0.05 \\
\hline DRB1*15:01 & 0.94 & $0.50-1.77$ & 0.84 & 0.84 & $0.56-1.27$ & 0.42 & 0.9 & $0.47-1.73$ & 0.75 \\
\hline DQA1*01:01 & 1.02 & $0.63-1.64$ & 0.94 & 0.64 & $0.46-0.89$ & 0.01 & 0.63 & $0.38-1.04$ & 0.07 \\
\hline DQA1*01:02 & 0.81 & $0.49-1.36$ & 0.43 & 0.83 & $0.60-1.14$ & 0.24 & 1.02 & $0.6-1.72$ & 0.96 \\
\hline DQA $1 * 01: 03$ & 1.02 & $0.54-1.91$ & 0.95 & 0.94 & $0.63-1.41$ & 0.77 & 0.93 & $0.49-1.75$ & 0.81 \\
\hline DQA1*02:01 & 0.92 & $0.54-1.57$ & 0.76 & 1.28 & $0.93-1.78$ & 0.13 & 1.4 & $0.81-2.41$ & 0.23 \\
\hline DQA1*03:01 & 0.61 & $0.34-1.11$ & 0.11 & 0.78 & $0.56-1.10$ & 0.16 & 1.28 & $0.7-2.34$ & 0.43 \\
\hline DQA1*05:01 & 1.50 & $1.00-2.27$ & 0.05 & 1.55 & $1.18-2.03$ & $1.76 \mathrm{E}-03$ & 1.03 & $0.68-1.56$ & 0.9 \\
\hline DQB1*02:01 & 1.62 & $1.05-2.50$ & 0.03 & 1.64 & $1.23-2.19$ & 8.48E-04 & 1.01 & $0.66-1.55$ & 0.97 \\
\hline DQB1*03:01 & 0.76 & $0.47-1.23$ & 0.27 & 1.12 & $0.85-1.49$ & 0.41 & 1.48 & $0.9-2.42$ & 0.12 \\
\hline DQB1*03:02 & 0.93 & $0.49-1.75$ & 0.82 & 0.86 & $0.58-1.27$ & 0.44 & 0.92 & $0.48-1.76$ & 0.81 \\
\hline DQB1*05:01 & 1.00 & $0.57-1.75$ & 1.00 & 0.72 & $0.49-1.05$ & 0.09 & 0.72 & $0.4-1.29$ & 0.26 \\
\hline DQB1*06:02 & 0.66 & $0.31-1.43$ & 0.30 & 1.01 & $0.66-1.55$ & 0.96 & 1.53 & $0.69-3.36$ & 0.29 \\
\hline DQB1*06:03 & 0.82 & $0.39-1.72$ & 0.60 & 1.09 & $0.71-1.67$ & 0.70 & 1.33 & $0.62-2.84$ & 0.46 \\
\hline DPA $1 * 01: 03$ & 1.24 & $0.80-1.93$ & 0.34 & 1.28 & $0.96-1.71$ & 0.09 & 1.04 & $0.66-1.62$ & 0.88 \\
\hline DPA $1 * 02: 01$ & 1.32 & $0.82-2.13$ & 0.25 & 1.41 & $1.03-1.93$ & 0.03 & 1.07 & $0.66-1.73$ & 0.79 \\
\hline $\mathrm{DPB} 1 * 02: 01$ & 1.46 & $0.90-2.37$ & 0.13 & 1.00 & $0.70-1.41$ & 0.98 & 0.68 & $0.41-1.12$ & 0.13 \\
\hline $\mathrm{DPB} 1 * 03: 01$ & 0.48 & $0.22-1.03$ & 0.06 & 0.75 & $0.50-1.11$ & 0.15 & 1.56 & $0.71-3.39$ & 0.27 \\
\hline DPB1*04:01 & 0.91 & $0.62-1.33$ & 0.62 & 0.91 & $0.72-1.16$ & 0.46 & 1.01 & $0.68-1.48$ & 0.98 \\
\hline DPB1*04:02 & 0.83 & $0.44-1.56$ & 0.56 & 0.96 & $0.66-1.39$ & 0.82 & 1.15 & $0.61-2.18$ & 0.67 \\
\hline
\end{tabular}

OR and 95\%CI estimated using polytomous logistic regression with adjustment on age, sex and genetic principal components.

The model includes 783 individuals $(381,67,335$ individuals with $0,1, \geq 5 \mathrm{sIgE}+$ respectively).

TABLE 4. Association between HLA class-II alleles and IgE recognition to major ragweed allergen Amb a 1, stratified by ragweed exposure levels

\begin{tabular}{|c|c|c|c|c|c|c|c|c|c|c|}
\hline \multirow[t]{2}{*}{ Allele } & \multicolumn{3}{|c|}{ All $(n=638)$} & \multicolumn{3}{|c|}{ Lyon $(n=145)$} & \multicolumn{3}{|c|}{$\begin{array}{l}\text { Paris-Marseille-Montpellier } \\
(\mathrm{n}=493)\end{array}$} & \multirow{2}{*}{$\begin{array}{l}\text { Interaction } \\
\text { p-value }\end{array}$} \\
\hline & OR & IC 95\% & p-value & OR & IC 95\% & p-value & OR & IC $95 \%$ & p-value & \\
\hline DQA1*05:01 & 1.94 & $1.17-3.19$ & 0.01 & 1.98 & $1.02-3.89$ & 0.04 & 1.07 & $0.29-3.29$ & 0.91 & 0.37 \\
\hline
\end{tabular}




\begin{tabular}{|c|c|c|c|c|c|c|c|c|c|c|}
\hline DQB1*02:01 & 1.92 & $1.14-3.17$ & 0.01 & 2.30 & $1.08-5.04$ & 0.03 & 1.49 & $0.41-4.55$ & 0.50 & 0.51 \\
\hline DRB1*03:01 & 2.06 & $1.04-3.83$ & 0.03 & 3.95 & $1.47-10.64$ & 0.006 & 1.52 & $0.22-6.39$ & 0.61 & 0.27 \\
\hline DQB1*05:01 & 0.21 & $0.04-0.69$ & 0.03 & - & - & - & 1.35 & $0.21-5.02$ & 0.70 & 0.98 \\
\hline DRB1*11:01 & 2.07 & $0.95-4.13$ & 0.05 & 1.57 & $0.54-4.05$ & 0.37 & 0.79 & $0.04-4.40$ & 0.83 & 0.59 \\
\hline DRB1*01:01 & 0.14 & $0.01-0.64$ & 0.05 & - & - & - & 0.80 & $0.04-3.86$ & 0.82 & 0.99 \\
\hline DQA1*01:01 & 0.52 & $0.20-1.11$ & 0.13 & 0.83 & $0.25-2.36$ & 0.74 & 0.46 & $0.03-2.24$ & 0.45 & 0.60 \\
\hline DQA1*02:01 & 1.54 & $0.83-2.72$ & 0.15 & 1.22 & $0.51-2.73$ & 0.64 & 2.31 & $0.63-7.14$ & 0.16 & 0.40 \\
\hline DQA1*01:02 & 0.58 & $0.24-1.19$ & 0.17 & 1.02 & $0.33-2.57$ & 0.96 & 0.39 & $0.02-1.97$ & 0.37 & 0.39 \\
\hline DPA $1 * 02: 01$ & 1.47 & $0.82-2.53$ & 0.171 & 0.75 & $0.32-1.61$ & 0.48 & 1.29 & $0.27-4.51$ & 0.72 & 0.48 \\
\hline DPB1*02:01 & 0.56 & $0.21-1.23$ & 0.19 & 0.34 & $0.08-1.04$ & 0.09 & 0.99 & $0.15-3.84$ & 0.99 & 0.30 \\
\hline DRB1*04:01 & 0.39 & $0.06-1.30$ & 0.20 & 0.34 & $0.02-1.90$ & 0.31 & 0.98 & $0.05-5.31$ & 0.98 & 0.49 \\
\hline DRB1*15:01 & 0.55 & $0.16-1.37$ & 0.25 & 0.86 & $0.18-3.02$ & 0.83 & - & - & - & 0.99 \\
\hline DPA $1 * 01: 03$ & 1.35 & $0.77-2.27$ & 0.28 & 0.79 & $0.35-1.67$ & 0.55 & 0.98 & $0.22-3.22$ & 0.97 & 0.74 \\
\hline DQA1*03:01 & 0.64 & $0.26-1.36$ & 0.29 & 0.44 & $0.12-1.22$ & 0.15 & 0.39 & $0.02-2.14$ & 0.38 & 0.99 \\
\hline DQB1*03:02 & 0.60 & $0.18-1.47$ & 0.32 & 0.31 & $0.05-1.13$ & 0.13 & - & - & - & 0.99 \\
\hline DPB1*04:02 & 1.37 & $0.65-2.60$ & 0.36 & 1.29 & $0.49-3.12$ & 0.58 & 2.23 & $0.50-7.45$ & 0.22 & 0.48 \\
\hline DQB1*03:01 & 1.26 & $0.71-2.13$ & 0.40 & 1.00 & $0.45-2.06$ & 1.00 & 1.01 & $0.23-3.23$ & 0.99 & 0.95 \\
\hline DQB1*06:02 & 0.65 & $0.19-1.63$ & 0.41 & 1.07 & $0.22-3.83$ & 0.93 & - & - & - & 0.99 \\
\hline DQA1*01:03 & 0.68 & $0.21-1.60$ & 0.44 & 0.25 & $0.01-1.24$ & 0.18 & 1.70 & $0.29-5.60$ & 0.45 & 0.12 \\
\hline DPB1*03:01 & 0.73 & $0.25-1.70$ & 0.52 & 0.54 & $0.09-1.03$ & 0.41 & 2.18 & $0.47-7.85$ & 0.26 & 0.18 \\
\hline DPB1*04:01 & 0.89 & $0.53-1.44$ & 0.63 & 1.58 & $0.84-2.97$ & 0.15 & 0.27 & $0.04-0.99$ & 0.09 & 0.03 \\
\hline DQB1*06:03 & 0.79 & $0.24-1.88$ & 0.65 & 0.30 & $0.02-1.48$ & 0.25 & 2.22 & $0.37-7.66$ & 0.27 & 0.12 \\
\hline
\end{tabular}

OR and $95 \%$ CI estimated using logistic regression with adjustment on age, sex and genetic principal components. 


\section{Figures legend}

\section{FIGURE 1. Flowchart}

EGEA: Epidemiological study of the Genetics and Environment of Asthma; FDR: False discovery rate; HLA: Human leukocyte antigen; IgE: Immunoglobulin E; MEDALL: Mechanisms of the Development of Allergy

FIGURE 2. Forest plot of statistically significant associations between HLA class-II alleles and respiratory allergen-specific IgE sensitization according to the asthma status (FDR $\leq \mathbf{0 . 0 5}$ ).

* the ORs could not be estimated because of the absence of sIgE-sensitized individuals for this respiratory allergen among participants without asthma.

OR: odds-ratio; CI: confidence interval

\section{Additional Files}

FIGURE E-1. Workflow

CCD: Cross-reactive carbohydrate determinant; HLA: Human leukocyte antigen; IgE: Immunoglobulin E; LD: linkage disequilibrium

TABLE E-1. Associations of respiratory allergen-specific IgE sensitization with HLA class-II alleles.

OR and 95\%CI estimated using mixed multiple regression adjusted for age, gender and genetic principal components and considering family as a random component. Models were performed assuming an additive genetic model.

TABLE E-2. Associations of respiratory allergen-specific IgE sensitization with HLA class-II alleles in the sub-population consisting in on member per family $(n=487)$.

OR and 95\%CI estimated using logistic regression adjusted for age, gender and genetic principal components. Models were performed assuming an additive genetic model 
TABLE E-3. Associations of respiratory allergen-specific IgE sensitization with HLA class-II alleles (SNP2HLA imputation), either under the dominant genetic model or and the codominant genetic model.

OR and 95\%CI estimated using logistic regression adjusted for age, gender and genetic principal components. Models were performed assuming an additive genetic model 
Figure 1

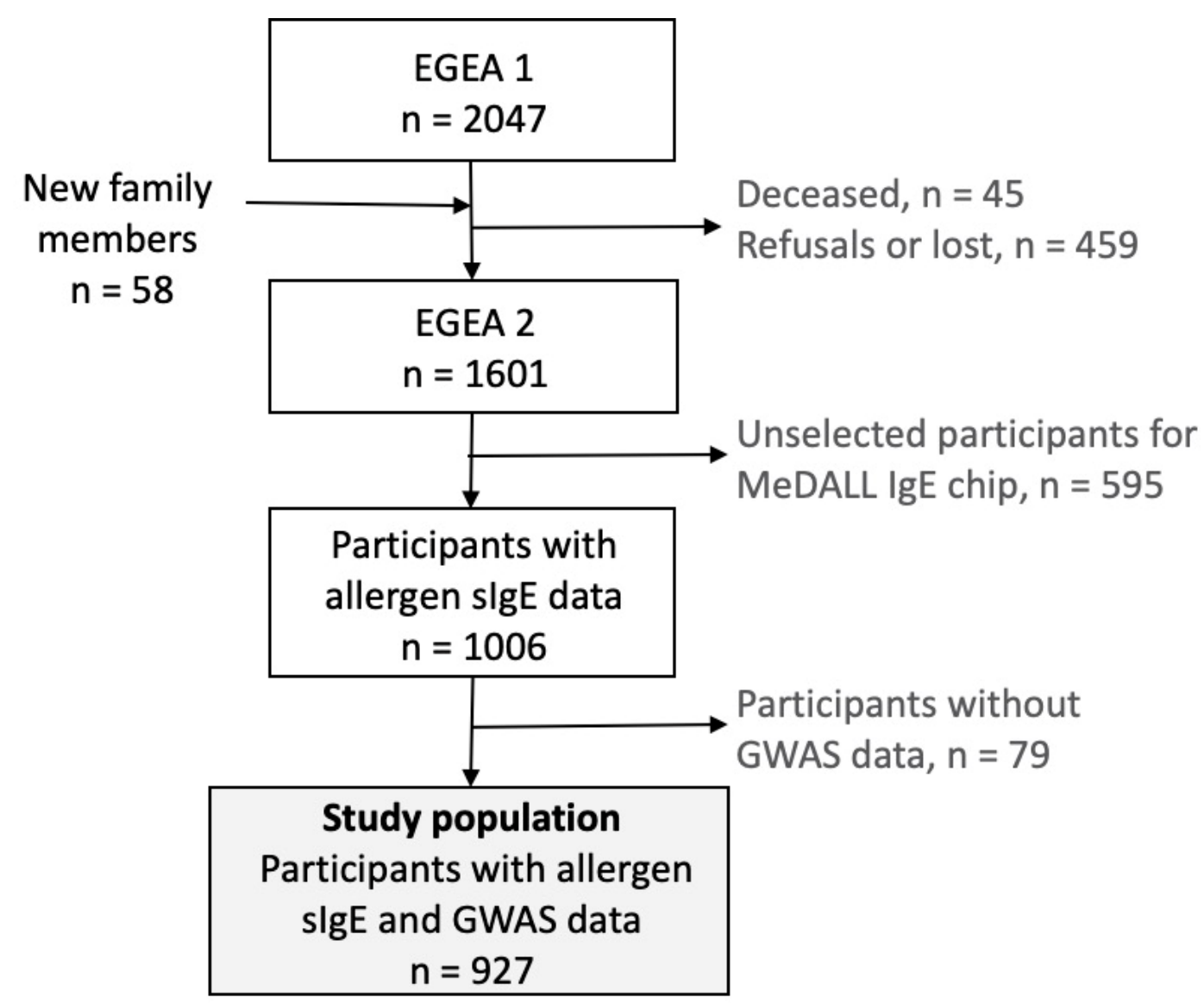


Figure 2
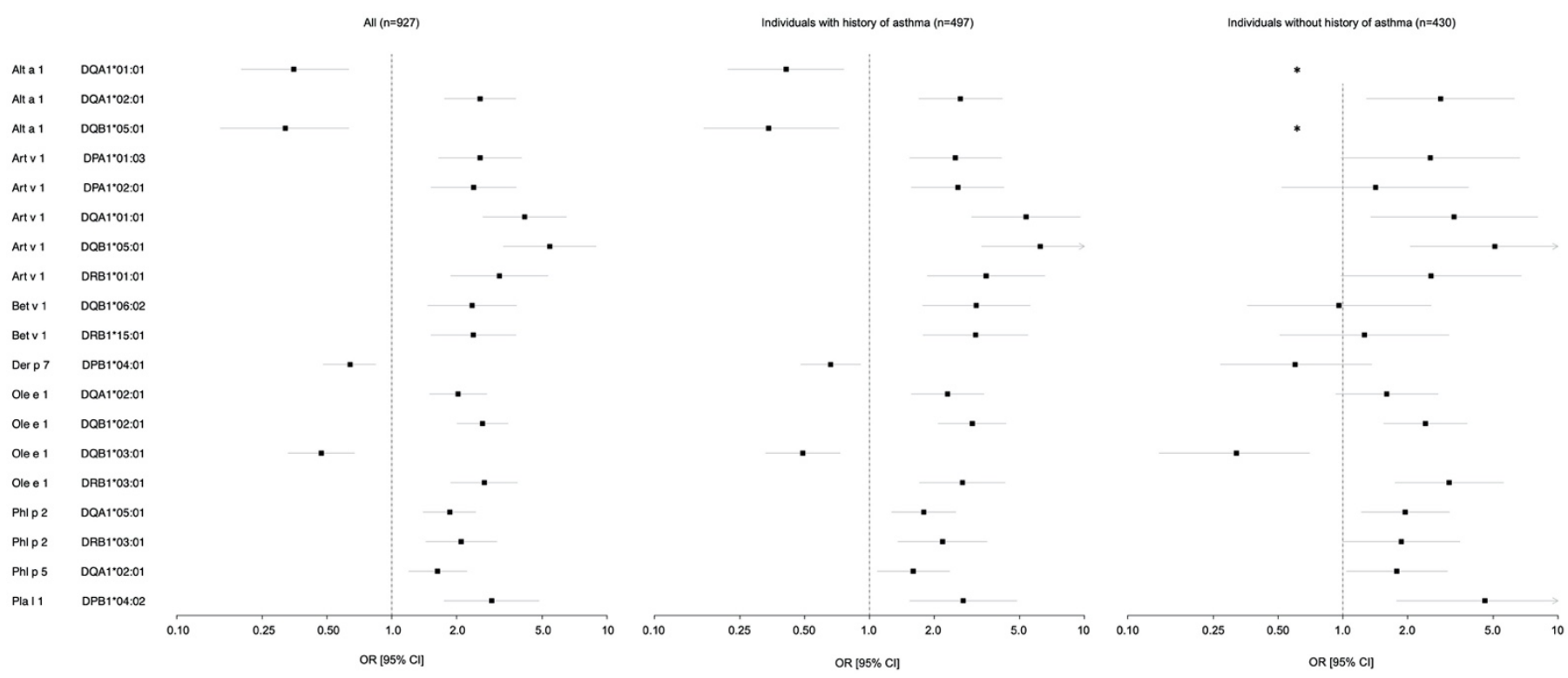